\title{
Previously reported placebo-response-associated variants do not predict patient outcomes in inflammatory disease Phase III trial placebo arms
}

\author{
Asher Haug-Baltzell, $\cdot$ Tushar R. Bhangale ${ }^{2,3} \cdot$ Diana Chang $^{2} \cdot$ Amy Dressen $^{2} \cdot$ Brian L. Yaspan $^{2} \cdot$ Ward Ortmann $^{4} \cdot$ \\ Matthew J. Brauer ${ }^{3} \cdot$ Julie Hunkapiller $^{2} \cdot$ Jens Reeder $^{3} \cdot$ Kiran Mukhyala $^{3} \cdot$ Karen T. Cuenco $^{2} \cdot$ Jennifer A. Tom $^{3}$. \\ Amy Cowgill ${ }^{3} \cdot$ Jan Vogel ${ }^{3} \cdot$ William F. Forrest ${ }^{3} \cdot$ Timothy W. Behrens $^{2} \cdot$ Robert R. Graham $^{2} \cdot$ Arthur Wuster $^{2,3}$
}

Received: 25 October 2017 / Revised: 29 January 2018 / Accepted: 31 January 2018 / Published online: 18 March 2018

(c) The Author(s) 2018. This article is published with open access

\begin{abstract}
In clinical trials, a placebo response refers to improvement in disease symptoms arising from the psychological effect of receiving a treatment rather than the actual treatment under investigation. Previous research has reported genomic variation associated with the likelihood of observing a placebo response, but these studies have been limited in scope and have not been validated. Here, we analyzed whole-genome sequencing data from 784 patients undergoing placebo treatment in Phase III Asthma or Rheumatoid Arthritis trials to assess the impact of previously reported variation on patient outcomes in the placebo arms and to identify novel variants associated with the placebo response. Contrary to expectations based on previous reports, we did not observe any statistically significant associations between genomic variants and placebo treatment outcome. Our findings suggest that the biological origin of the placebo response is complex and likely to be variable between disease areas.
\end{abstract}

\section{Introduction}

In clinical trials, the placebo response refers to a patient's improvement in disease symptoms arising from the psychological effect of receiving a treatment rather than the actual treatment under investigation [1]. This improvement can be actual or perceived, and can encompass diverse causes such as the natural waxing-waning of disease

Electronic supplementary material The online version of this article (https://doi.org/10.1038/s41435-018-0018-z) contains supplementary material, which is available to authorized users.

Arthur Wuster

wustera@gene.com

1 Genetics Interdisciplinary Group, University of Arizona, Tucson, AZ 85719, USA

2 Department of Human Genetics, Genentech Inc, South San Francisco 94080 CA, USA

3 Department of Bioinformatics and Computational Biology, Genentech Inc, South San Francisco 94080 CA, USA

4 Biosample and Depository Management, Genentech Inc, South San Francisco, CA 94080, USA symptoms, regression to the mean, response to concurrent medications, spontaneous remission, or a biologically mediated true placebo response [2, 3]. Considering that successful outcomes of placebo-controlled randomized trials are achieved by demonstrating a greater improvement in treatment arms than the placebo arms, and the primary cause of Phase III trial failure is a demonstrated lack of efficacy, it is clear that a solid understanding of the placebo response is essential for maximizing the chances of success of such trials [4, 5]. This is particularly important considering the current downward trend in clinical trial success rate, and a corresponding increase in cost per new drug approved that has, by some estimates, reached $\$ 2$ billion [6].

One way to improve the chances of clinical trial success is by improving the trial design. A fundamental assumption underlying the placebo-controlled trial design is that placebo treatment serves as an effective and unbiased control, and thus failure to outperform a placebo arm is indicative of a lack of drug efficacy. However, extensive research highlighting the powerful and biological nature of the placebo effect calls this assumption into question [2, 7-9]. Neuroimaging studies have demonstrated that the placebo response can, at least in certain cases, be biologically mediated 


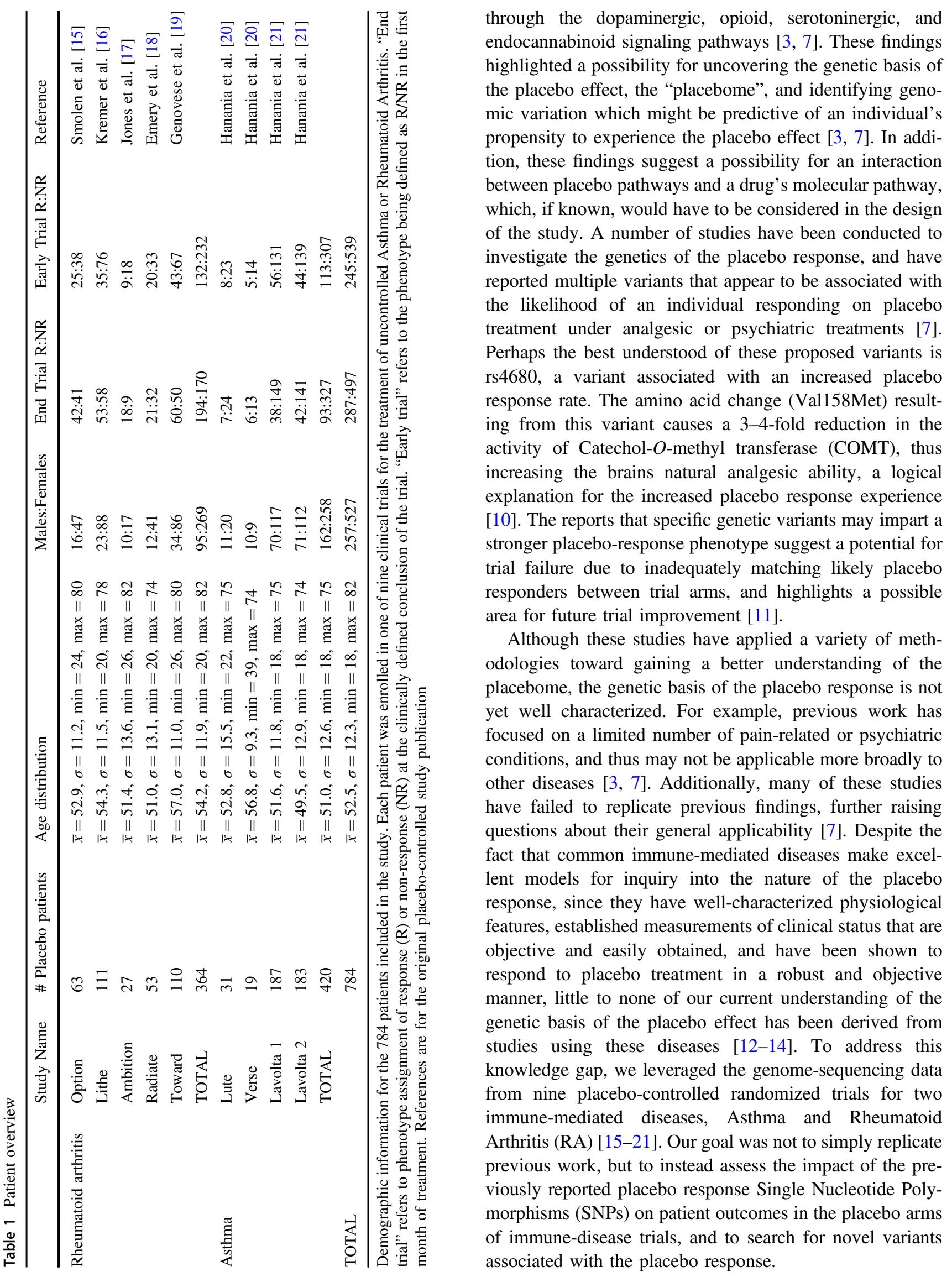

through the dopaminergic, opioid, serotoninergic, and endocannabinoid signaling pathways $[3,7]$. These findings highlighted a possibility for uncovering the genetic basis of the placebo effect, the "placebome", and identifying genomic variation which might be predictive of an individual's propensity to experience the placebo effect $[3,7]$. In addition, these findings suggest a possibility for an interaction between placebo pathways and a drug's molecular pathway, which, if known, would have to be considered in the design of the study. A number of studies have been conducted to investigate the genetics of the placebo response, and have reported multiple variants that appear to be associated with the likelihood of an individual responding on placebo treatment under analgesic or psychiatric treatments [7]. Perhaps the best understood of these proposed variants is rs4680, a variant associated with an increased placebo response rate. The amino acid change (Val158Met) resulting from this variant causes a 3-4-fold reduction in the activity of Catechol- $O$-methyl transferase (COMT), thus increasing the brains natural analgesic ability, a logical [10]. The reports that specific genetic varants may impart a stronger placebo-response phenotype suggest a potential for trial failure due to inadequately matching likely placebo responders between trial arms, and highlights a possible area for future trial improvement [11].

Although these studies have applied a variety of methodologies toward gaining a better understanding of the placebome, the genetic basis of the placebo response is not yet well characterized. For example, previous work has focused on a limited number of pain-related or psychiatric conditions, and thus may not be applicable more broadly to or diseases [3, 7]. Additionally, many of these studies ave failed to replicate previous findings, further raising questions about their general applicability [7]. Despite the fact that common immune-mediated diseases make excelnt models for inquiry into the nature of the placebo esponse, since they have well-characterized physiological features, established measurements of clinical status that are objective and easily obtained, and have been shown to respond to placebo treatment in a robust and objective manner, little to none of our current understanding of the genetic basis of the placebo effect has been derived from studies using these diseases [12-14]. To address this knowledge gap, we leveraged the genome-sequencing data from nine placebo-controlled randomized trials for two immune-mediated diseases, Asthma and Rheumatoid Arthritis (RA) [15-21]. Our goal was not to simply replicate previous work, but to instead assess the impact of the previously reported placebo response Single Nucleotide Polymorphisms (SNPs) on patient outcomes in the placebo arms associated with the placebo response. 


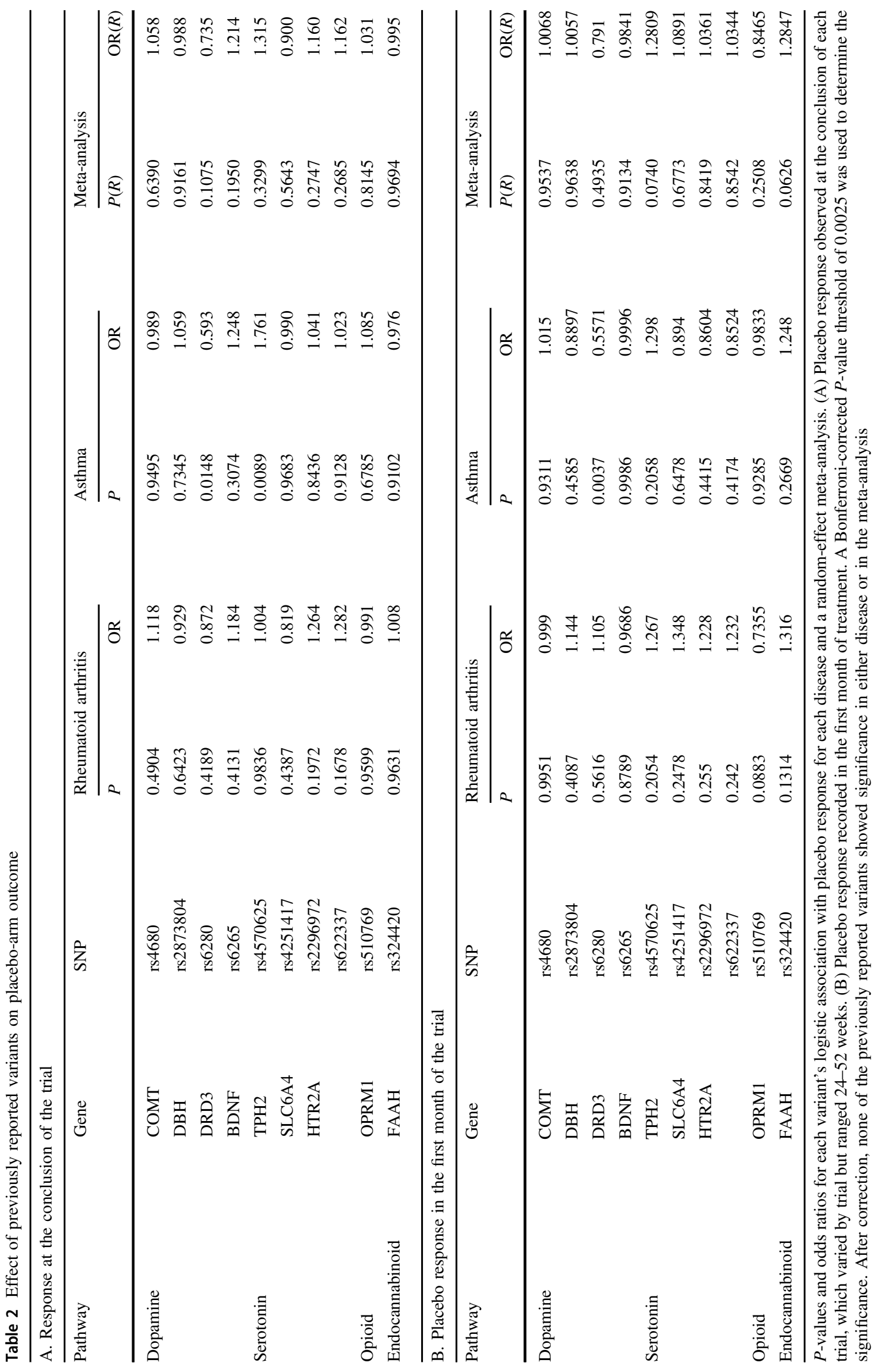


Measuring the placebo response can be challenging, especially when attempting to compare multiple different diseases. In this study, we chose to primarily focus on patient-reported wellness outcomes as reported in qualityof-life (QOL) questionnaires collected at the preliminary screening and regularly throughout the trial progression. Using these measurements, we classified patients dichotomously as either placebo "responders" (those who showed an improvement in QOL) and placebo "non-responders" (those who showed no improvement or a worsening of QOL). The choice of using change in QOL as the primary outcome measure was deemed necessary and appropriate for two major reasons. First, using changes in QOL provided a uniform method of measuring patient outcomes between multiple independent trials treating two different diseases. This enabled us to both conduct meta-analyses between disease areas and increase our statistical confidence in the conclusions. Secondly, the placebo response is more pronounced in subjective symptom measurements, such as those recorded in QOL questionnaires than in objective measurements $[2,22]$, so we expected to see greater differentiation between placebo responders and non-responders in these measurements. However, we also conducted secondary analyses using physiological clinical endpoints to validate the applicability of the findings to the trial design. For RA trials, the physiological clinical endpoint was an improvement of at least $20 \%$ in the American College of Rheumatology score (ACR20) and for Asthma trials, an above median change in the Forced Expiratory Volume $\left(\mathrm{FEV}_{1}\right)$, a measure of lung volume frequently recorded in Asthma trials.

\section{Results and discussion}

Our primary aim was to investigate the effect of previously reported placebo-associated genetic variants on patient outcomes in the placebo arms of Phase III trials for Asthma and RA. To address this question, we analyzed 784 patients from nine Phase III trials. Of those, 364 had been diagnosed with active RA and participated in one of five Phase III trials investigating Tocilizumab, a humanized monoclonal antibody targeting the Interleukin 6 receptor (IL6R). The other 420 patients were diagnosed with moderate to severe Asthma and participated in one of four trials investigating Lebrikizumab, a humanized monoclonal antibody targeting Interleukin 13 (IL13) (Table 1). Because it has been demonstrated that the placebo response is most evident in patient-reported assessments of their symptoms (e.g., QOL questionnaires) rather than in clinician-measured outcomes such as ACR20 or $\operatorname{FEV}_{1}[2,22]$, we defined placebo response as a patient-reported improvement of symptoms in disease-specific QOL questionnaires. Furthermore, using the QOL questionnaires for phenotype definitions enabled direct comparisons between trials and between disease areas.

We initially focused on the changes in symptoms relative to the baseline at the conclusion of each trial to maximize the relevancy of our findings to trial design. Using a logistic regression approach for identifying the genetic associations while accounting for potentially confounding variables, followed by random-effect model meta-analysis to combine our results from the two disease areas, we tested each previously reported autosomal placebo-associated variant for significant association with patients who reported an improvement of symptoms versus no change or worsening of symptoms (see "Patients and methods" section for discussion of phenotype assignment). We began our analyses by testing the 10 previously reported variants in autosomal genes involved in the four hypothesized placebo-implicated pathways (Table 2a). We were well powered to detect the associations in these variants with strong effects (Power = 0.86 , assuming an effect size of 1.7 , a minor allele frequency of c. $45 \%$, which is comparable to rs 4680 , and the commonly accepted placebo response rate of 35\% [23-25]). After Bonferroni correction for multiple hypothesis testing, none of the previously reported placebo-associated variants, including the well-characterized SNP rs4680, were significantly associated with patient-reported improvements at the conclusion of each trial in RA, Asthma, or a metaanalysis. In fact, most variants were well balanced between responders/non-responders (Supplemental Table 1). In the five RA trials, all variants shared similar allelic frequencies between the case/control groups. In Asthma, only two variants, rs4570625 from THP2 $(P=0.009, \mathrm{OR}=1.761)$ and rs6280 from DRD3 $(P=0.015, \mathrm{OR}=0.5933)$, showed a moderate, yet still statistically insignificant, difference. Notably, consistent with previous findings, Asthma patients homozygous for $\mathrm{T} / \mathrm{T}$ (Ser/Ser) at rs6280 were enriched in the placebo responders group (59\% vs. $47 \%$ ), although this was not observed in RA patients [26]. On the other hand, Asthma patients homozygous for $\mathrm{G} / \mathrm{G}$ at rs4570625 were more common in the non-responders group, an effect direction that is opposite to the previous findings [27]. Combined with the fact that neither of these two variants showed even moderate significance under a random-effect meta-analysis model or under association testing for physiological clinical endpoints (Supplemental Table 2), we conclude that these variants do not significantly impact the placebo response in these trials. 
Since we were unable to validate the previously reported variants, we hypothesized that the placebo effect might be a short-lived response and symptoms could return to normal or worsen over the long timeframe of Phase III trials (44-52 weeks). To test this hypothesis, we re-analyzed each previously reported variant, but redefined "placebo response" as a patient-reported improvement in symptoms during the first 4 weeks of the trial (percent change from baseline). Again, none of the previously reported variants showed a significant association with symptom improvement in the placebo arm (Table 2b, Supplemental Table 2). In Asthma, the two variants showing the lowest $P$-values in the later placebo response again showed the lowest $P$-values and same effect direction, although they still remained statistically insignificant. Interestingly, in RA, the OPRMI variant rs510769 showed a sharp decrease in $P$-value relative to the later time point, from 0.96 to 0.08 , while maintaining an effect in the same direction. Taken together, these results suggest that the previously reported placeboresponse-associated variants may not have a strong effect on patient outcomes in the placebo arms of immune-related disease trials.

Because the placebo-associated variants identified in studies investigating placebo treatments in pain and psychiatric disorders did not appear significantly associated with placebo-arm outcomes in immune-mediated disease trials, we sought to identify any novel variants that might have a strong effect under these disease conditions. We limited this analysis to patient response at the conclusion of each trial, as this timepoint is the most relevant to improving trial outcomes. We limited our analysis to common autosomal SNPs (MAF $>5 \%$ ), and after QC and filtering, we tested 5,630,720 SNPs for RA, and 4,918,428 for Asthma. For each disease, we tested these SNPs for association with a placebo response while controlling for the potentially confounding effects of age, sex, population structure, and baseline disease severity. We observed no evidence of genomic inflation of the test statistic (inflation factors 1.047 and 1.024 for RA and Asthma, respectively) suggesting that the likely confounding factors were well accounted for. We also performed a random-effect metaanalysis of these results, where we analyzed the $4,697,728$ shared SNPs. Although the study was well powered to detect common variants with strong effects (Power $=0.86$, assuming an effect size of 1.7, a minor allele frequency of c. $45 \%$, which is comparable to rs 4680 , and the commonly accepted placebo response rate of 35\% [2325]), we were unable to detect any genome-wide significant hits (Supplemental Fig. 1). In RA, the most significant hit was a haplotype block tagged by $\operatorname{rs} 2613713(P=1.426 \mathrm{e}$ $-06, \mathrm{OR}=2.363$ ), which lies in an intergenic region on chromosome 8 (p21.3). However, this block became convincingly insignificant under the meta-analysis $\left(P_{\mathrm{R}}=\right.$ $0.15, \mathrm{OR}_{\mathrm{R}}=1.656$ ). In Asthma patients, the most significant hit was rs6694886, an intronic $\mathrm{G}>\mathrm{T}$ variant in CAMTA1 (calmodulin binding transcription activator 1) on chromosome $1(P=2.529 \mathrm{e}-06, \mathrm{OR}=2.448)$. However, like with the most significant hit in RA, evidence vanished under the meta-analysis, dropping to $P_{\mathrm{R}}=0.5948$ with $\mathrm{OR}_{\mathrm{R}}=1.362$. Because none of the limited "suggestive" variants were genome-wide significant $(P<10 \mathrm{e}-8)$, and because they lost statistical evidence under a random-effect meta-analysis, we are not confident that these are true placebo-associated variants. Rather, our results suggest that individual gene variants do not strongly affect the placebo response. However, it is important to note that due to the small sample size in this study, we are unable to rule out minor effects of single variants, or any effect that might arise from pathway-wide burdens.

In summary, our findings suggest that the biological origin of the placebo response is complex and likely variable between disease areas, and care must be taken when generalizing findings regarding the genetic causes of the placebo effect. Although previous studies have identified variants that influence the likelihood of response to placebo treatment for pain-related and psychiatric conditions, these variants appear to have very little, if any, effect on the experiences of placebo-arm patients in Phase III trials for immune-mediated diseases. Furthermore, although our study was moderately well powered, we were unable to detect any genome-wide significant variations between placebo responders and non-responders, supporting the conclusion that although genetics may play a role in the placebo effect, the role of genomic variation is small and likely overpowered by other, possibly non-genetic factors. Similar to previous studies, we are limited in drawing full conclusions by the lack of a no-treatment control. Unfortunately, however, we see little option for addressing this shortfall, as such a control group is not possible for diseases with existing acceptable treatment options.

Our study highlights the need for further research into the genetic basis of the placebo effect. It is likely that, like other complex traits, the placebo response is modulated by multiple variants with a relatively small effect each. In order to identify those variants, a larger sample size is likely to be required. We believe costs associated with obtaining the sequencing data would be justified, because a solid understanding of the variation affecting response under placebo treatment would allow its confounding influence could be accounted for in trial design, thus potentially leading to more efficient, less costly trials with higher success rates. 


\section{Patients and methods}

This analysis was performed on 784 non-related European patients from the placebo arms of nine clinical trials for RA and Asthma. Patients were 18-82 years old and the male: female ratio was 257:527 (Table 1). Genomic DNA was extracted from patients' blood samples using the DNA Blood400 kit (Chemagic) and eluted in $50 \mu \mathrm{L}$ Elution Buffer (EB, Qiagen). It was then sheared (Covaris LE220) and sequencing libraries were prepared using the TruSeq Nano DNA HT kit (Illumina Inc.). The whole-genome sequencing target coverage was $30 \times$. Libraries were sequenced as 150-bp paired-end reads on the Illumina HiSeqX sequencer. Reads were mapped to hg38 using BWA-MEM [28] and variant calling was performed using GATK best practices [29]. Variant calling was performed using GATK HaplotypeCaller to generate sample level variant calls. The samples were then jointly genotyped separately for Asthma and RA samples, resulting in one VCF file for each disease area. To assure that only the most high-confidence variants are included in the association analysis, we set genotypes whose genotype quality was less than 20 to missing. We also removed variants with a missingness of more than $1 \%$. Because our focus was on previously reported common variants and because of the limited size of our sample set, we chose to not include variants with an allele frequency of less than $5 \%$ in subsequent analyses.

The population structure was determined for patient cohorts from the joint genotyping SNPs from each disease (analyzed separately) using the GENESIS R package (version 2.6.1) [30]. GENESIS uses the PC-AiR method to determine population structure, a PrincipalComponents-based analysis that assesses cryptic relatedness from genome-wide sets of SNPs while accounting for potentially confounding relatedness [30]. GENESIS was also used to verify previous relatedness estimations, ensuring that all individuals included in the analyses were unrelated.

Each individual was determined to be a responder or a non-responder at two timepoints. We defined responders as those patients who reported improvement in disease severity, as assessed by the percent change from baseline in the appropriate QOL questionnaire, whereas non-responders were those patients who reported no change or a worsening of symptoms. For consistent comparison between disease areas, no minimum improvement cutoff was enforced. In other words, responders may display any amount of positive change in symptoms, even if minor. For RA, this response was recorded in the Health Assessment Questionnaire (HAQ), and for Asthma, the Asthma Quality of Life Questionnaire (AQLQ). A placebo response greater than zero was observed in all trials, and in most cases the placebo response exceeded the minimal clinically important difference (MCID). For example, the AQLQ changes for the placebo arms of LAVOLTA 1 and 2 were 0.78 and 0.80 , respectively, which exceed the MCID of $0.5^{18}$.

Using QOL questionnaires provided two benefits over other recorded values: first, placebo responses are most evident in patient-reported symptom assessments [2, 22], and second it represents the most consistent measurement of outcome between different trials and disease areas. In the cases where a value was absent, we used a last observation carried forward (LOCF) approach to fill in missing values. This approach was most notably applied in the Lute and Verse trials, as the early discontinuation of these trials resulted in those patients enrolled later missing data at the specified endpoint. Each individual was assigned two phenotypes based on timeframe of comparison to baseline, where an early response refers to a reported improvement in symptoms in the first month of the trial (assessed at week 4), and late responders were those who reported an improvement at the conclusion of the trial (week 24 for RA trials and week 52 for Asthma trials).

To identify the variants associated with placebo response, we used PLINK (1.90b4.4) to perform logistic regression genetic association analysis contrasting placebo responders and non-responders [31-33]. Age, sex, baseline disease severity (baseline clinical measure of interest, e.g., AQLQ/HAQ), population structure (first five principal components from GENESIS), and trial (as dummy variable) were included as covariates to account for confounding influences such as imbalance and regression to the mean. Our approach assumed additive genetic effects, i.e., one alternate allele has a bigger effect than none, and that two have a bigger effect than one. In total, three rounds of association studies were performed; first, only the previously reported placebo-associated autosomal SNPs were tested at two time-points (conclusion of the trial and first month of treatment), and later a genome-wide set of common (MAF $>5 \%$ ) autosomal SNPs were investigated (only at the conclusion of the trial). Association for each disease was performed separately. Following disease-specific association analysis, a meta-analysis (--meta flag in PLINK) combining the two diseases areas was performed using the same version of PLINK to determine those variants with generalized placebo-response-associated phenotypes [34]. Because placebo response rates varied between diseases, only the more conservative random-effect model was interpreted. 
Acknowledgements We thank all of our Genentech colleagues involved in the Human Genetics Initiative, with particular thanks to Suresh Selvaraj, Delphine Lagarde, and Biosample Management Repository; Slaton Lipscomb, Craig Amundsen, and Pharmainformatics; Kelly Mewes, Alex Hughes, and Biometrics; as well as David Choy for help with the Asthma data, and Dheeraj Malhotra from Pharma Research and Early Development at Roche for comments on the project.

\section{Compliance with ethical standards}

Conflict of interest All authors except AHB are employees of Genentech Inc. Stock options form part of the company's remuneration package.

Open Access This article is licensed under a Creative Commons Attribution 4.0 International License, which permits use, sharing, adaptation, distribution and reproduction in any medium or format, as long as you give appropriate credit to the original author(s) and the source, provide a link to the Creative Commons license, and indicate if changes were made. The images or other third party material in this article are included in the article's Creative Commons license, unless indicated otherwise in a credit line to the material. If material is not included in the article's Creative Commons license and your intended use is not permitted by statutory regulation or exceeds the permitted use, you will need to obtain permission directly from the copyright holder. To view a copy of this license, visit http://creativecommons. org/licenses/by/4.0/.

\section{References}

1. Miller FG, Kaptchuk TJ. The power of context: reconceptualizing the placebo effect. J R Soc Med. 2008;101:222-5.

2. Finniss DG, Kaptchuk TJ, Miller F, Benedetti F. Biological, clinical, and ethical advances of placebo effects. Lancet. 2010;375:686-95.

3. Colagiuri B, Schenk LA, Kessler MD, Dorsey SG, Colloca L. The placebo effect: from concepts to genes. Neuroscience. 2015;307:171-90.

4. Grignolo A, Pretorius S. Phase III trial failures: costly, but preventable. Appl Clin Trials. 2016;25. http://www.appliedclinica ltrialsonline.com/phase-iii-trial-failures-costly-preventable.

5. Scannell JW, Blanckley A, Boldon H, Warrington B. Diagnosing the decline in pharmaceutical R\&D efficiency. Nat Rev Drug Discov. 2012;11:191-200.

6. Mullin R. Tufts study finds big rise in cost of drug development I chemical \& engineering news. Chem Eng News. 2014. http://cen. acs.org/articles/92/web/2014/11/Tufts-Study-Finds-Big-Rise. html. Accessed 7 Aug 2017.

7. Hall KT, Loscalzo J, Kaptchuk TJ. Genetics and the placebo effect: the placebome. Trends Mol Med. 2015;21:285-94.

8. Kaptchuk TJ, Miller FG. Placebo effects in medicine. N Engl J Med. 2015;373:8-9.

9. Holmes RD, Tiwari AK, Kennedy JL. Mechanisms of the placebo effect in pain and psychiatric disorders. Pharmacogenomics J. 2016;16:491-500.

10. Hall KT, Lembo AJ, Kirsch I, Ziogas DC, Douaiher J, Jensen KB, et al. Catechol-O-methyltransferase val158met polymorphism predicts placebo effect in irritable bowel syndrome. PLoS ONE. 2012;7:e48135.

11. Servick K. Outsmarting the placebo effect. Science. 2014;345:1446-7.
12. Butler C, Steptoe A. Placebo responses: an experimental study of psychophysiological processes in asthmatic volunteers. Br J Clin Psychol. 1986;25:173-83. (Pt 3)

13. Kemeny ME, Rosenwasser LJ, Panettieri RA, Rose RM, BergSmith SM, Kline JN. Placebo response in asthma: a robust and objective phenomenon. J Allergy Clin Immunol. 2007;119:1375-81.

14. Abdullah N. Placebo effect in the treatment of rheumatoid arthritis: a systematic review and meta-analysis of randomized controlled trials. Ann Rheum Dis. 2015;74:263-263.

15. Smolen JS, Beaulieu A, Rubbert-Roth A, Ramos-Remus C, Rovensky J, Alecock E, et al. Effect of interleukin-6 receptor inhibition with tocilizumab in patients with rheumatoid arthritis (OPTION study): a double-blind, placebo-controlled, randomised trial. Lancet. 2008;371:987-97.

16. Kremer JM, Blanco R, Brzosko M, Burgos-Vargas R, Halland A$\mathrm{M}$, Vernon E, et al. Tocilizumab inhibits structural joint damage in rheumatoid arthritis patients with inadequate responses to methotrexate: results from the double-blind treatment phase of a randomized placebo-controlled trial of tocilizumab safety and prevention of structural joint damage at one year. Arthritis Rheum. 2011;63:609-21.

17. Jones G, Sebba A, Gu J, Lowenstein MB, Calvo A, Gomez-Reino $\mathrm{JJ}$, et al. Comparison of tocilizumab monotherapy versus methotrexate monotherapy in patients with moderate to severe rheumatoid arthritis: the AMBITION study. Ann Rheum Dis. 2010;69:88-96.

18. Emery P, Keystone E, Tony HP, Cantagrel A, van Vollenhoven R, Sanchez A, et al. IL-6 receptor inhibition with tocilizumab improves treatment outcomes in patients with rheumatoid arthritis refractory to anti-tumour necrosis factor biologicals: results from a 24-week multicentre randomised placebo-controlled trial. Ann Rheum Dis. 2008;67:1516-23.

19. Genovese MC, McKay JD, Nasonov EL, Mysler EF, da Silva NA, Alecock E, et al. Interleukin-6 receptor inhibition with tocilizumab reduces disease activity in rheumatoid arthritis with inadequate response to disease-modifying antirheumatic drugs: the tocilizumab in combination with traditional disease-modifying antirheumatic drug therapy study. Arthritis Rheum. 2008;58:2968-80.

20. Hanania NA, Noonan M, Corren J, Korenblat P, Zheng Y, Fischer $\mathrm{SK}$, et al. Lebrikizumab in moderate-to-severe asthma: pooled data from two randomised placebo-controlled studies. Thorax. 2015;70:748-56.

21. Hanania NA, Korenblat P, Chapman KR, Bateman ED, Kopecky $\mathrm{P}$, Paggiaro $\mathrm{P}$, et al. Efficacy and safety of lebrikizumab in patients with uncontrolled asthma (LAVOLTA I and LAVOLTA II): replicate, phase 3 , randomised, double-blind, placebo-controlled trials. Lancet Respir Med. 2016;4:781-96.

22. Wechsler ME, Kelley JM, Boyd IOE, Dutile S, Marigowda G, Kirsch I, et al. Active albuterol or placebo, sham acupuncture, or no intervention in asthma. N Engl J Med. 2011;365:119-26.

23. Beecher HK. The powerful placebo. J Am Med Assoc. 1955;159:1602-6.

24. Johnson JL. GAS Power Calculator. 2017. http://csg.sph.umich. edu/abecasis/cats/gas_power_calculator/reference.html. Accessed 9 Aug 2017.

25. Skol AD, Scott LJ, Abecasis GR, Boehnke M. Joint analysis is more efficient than replication-based analysis for two-stage genome-wide association studies. Nat Genet. 2006;38:209-13.

26. Bhathena A, Wang Y, Kraft JB, Idler KB, Abel SJ, Holley-Shanks $\mathrm{RR}$, et al. Association of dopamine-related genetic loci to dopamine D3 receptor antagonist ABT-925 clinical response. Transl Psychiatry. 2013;3:e245. 
27. Furmark T, Appel L, Henningsson S, Ahs F, Faria V, Linnman C, et al. A link between serotonin-related gene polymorphisms, amygdala activity, and placebo-induced relief from social anxiety. J Neurosci. 2008;28:13066-74.

28. Li H. Aligning sequence reads, clone sequences and assembly contigs with BWA-MEM. ArXiv13033997 Q-Bio. 2013. http://a rxiv.org/abs/1303.3997.

29. GATK I Best Practices. https://software.broadinstitute.org/gatk/ best-practices/. Accessed 6 Sep 2017.

30. Conomos MP, Thornton T, Gogarten SM. GENetic EStimation and Inference in Structured samples (GENESIS): statistical methods for analyzing genetic data from samples with population structure and/or relatedness. 2017. http://bioconductor.org/packa ges/GENESIS/.
31. Purcell S, Chang C. PLINK v.1.90b4.4. www.cog-genomics.org/ plink/1.9/.

32. Chang CC, Chow CC, Tellier LC, Vattikuti S, Purcell SM, Lee JJ. Second-generation PLINK: rising to the challenge of larger and richer datasets. Gigascience. 2015;4:7.

33. Hill A, Loh P-R, Bharadwaj RB, Pons P, Shang J, Guinan E, et al. Stepwise distributed open innovation contests for software development: acceleration of genome-wide association analysis. Gigascience. 2017;6:1-10.

34. Willer CJ, Li Y, Abecasis GR. METAL: fast and efficient metaanalysis of genomewide association scans. Bioinformatics. 2010;26:2190-1. 